\title{
A CLASS OF MANIFOLDS COVERED BY EUCLIDEAN SPACE
}

\author{
J. W. MAXWELL ${ }^{1}$
}

ABSTRACT. The following is the main result:

Theorem 1. Suppose $W^{n}$ is a PL manifold which has homotopy type $K(\Pi, 1), W$ has one end $\infty, \pi_{1}$ is essentially constant at $\infty$, and the induced homomorphism $\pi_{1}(\infty) \rightarrow \pi_{1}(W)$ is an isomorphism. Then the universal cover of $W$ is $P L$ homomorphic to $R^{n}$ provided $n \geq 5$.

1. Introduction. Let $W^{n}$ denote a topological manifold of dimension $n$. $W$ is said to have homotopy type $K(\Pi, 1)$ provided $\pi_{1}(W) \simeq \Pi$ and the universal cover $W$ of $W$ is contractible. A natural question is: Under what conditions is $\tilde{W}$ homeomorphic to $R^{n}$ ? For $n=1,2$, no additional assumptions are necessary since $\widetilde{W}$ contractible implies it is homeomorphic to $R^{n}$. For $n \geq 3$, the existence of contractible manifolds $W$ not homeomorphic to $R^{n}$ makes some restrictions likely. In the next section an example is given of a 3-dimen sional, open (noncompact and empty boundary) manifold of homotopy type $K(\Pi, 1)$ whose universal cover is not homeomorphic to $R^{3}$. It is a long-standing conjecture that the universal cover of a closed (compact and empty boundary) manifold $W^{n}$ of homotopy type $K(\Pi, 1), \Pi$ an infinite group, is homeomorphic to $R^{n}$. Some partial results to this conjecture in the case $n \geq 5$ can be found (without proof) in [3]. In [7] Waldhausen gives sufficient conditions for the universal cover of a closed 3-manifold of homotopy type $K(\Pi, 1)$ to be homeomorphic to $R^{3}$. The author knows of no counterexample to this conjecture.

This paper is concerned with open $K(\Pi, 1)$ manifolds which admit a piecewise linear structure. For the remainder of this paper let $W^{n}$ denote such a manifold, and suppose further that $W$ has a single end which is denoted by $\infty$. An inverse sequence of groups

Received by the editors October 3, 1973 and, in revised form, November 8, 1973. AMS (MOS) subject classifications (1970). Primary 57C25; Secondary 57C 30.

Key words and phrases. Homotopy type $K(\pi, 1)$, universal cover, euclidean space, end.

${ }^{1}$ Research partially supported by the College of Arts and Sciences Office of Research and Graduate Studies, Oklahoma State University. 


$$
G_{1} \stackrel{f_{1}}{\longleftarrow} G_{2} \stackrel{f_{2}}{\longleftarrow} G_{3} \stackrel{f_{3}}{\longleftarrow} \cdots
$$

is said to be essentially constant if there exists a subsequence

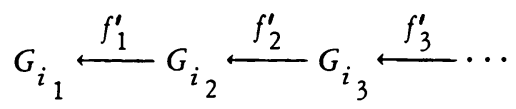

so that isomorphisms $\operatorname{Im}\left(f_{1}^{\prime}\right) \cong \operatorname{Im}\left(f_{2}^{\prime}\right) \cong \operatorname{Im}\left(f_{3}^{\prime}\right)$ are induced. Following [5], we say that $\pi_{1}$ is essentially constant at $\infty$ if for a sequence $K_{1} \subset K_{2} \subset K_{3}$ of compacta with $W=\bigcup_{\text {int }} K_{i}$, the sequence induced by inclusion

$$
\text { S: } \pi_{1}\left(W-K_{1}\right) \leftarrow \pi_{1}\left(W-K_{2}\right) \leftarrow \pi_{1}\left(W-K_{3}\right) \leftarrow \cdots
$$

is essentially constant. The choice of $\left\{K_{i}\right\}$, of base points, and of connecting base paths used to define $\mathcal{S}$ does not affect this property. Also $\pi_{1}(\infty)$ $=$ proj $\lim (\mathcal{S})$ proves to be independent of these choices up to isomorphism in a preferred conjugacy class; and $\pi_{1}\left(W-K_{i}\right) \rightarrow \pi_{1}(W)$, for large $i$, induces a homomorphism $\pi_{1}(\infty) \rightarrow \pi_{1}(W)$ which is again determined within a conjugacy class. For a verification of these facts see [6].

Let $M$ denote a compact manifold with nonempty boundary of homotopy type $K(\Pi, 1)$. If $\pi_{1}(\partial M)$ is isomorphic by inclusion to $\pi_{1}(M)$, then $W=$ int $M$ is an open manifold with one end $\infty, \pi_{1}$ is essentially constant at $\infty$, and $\pi_{1}(\infty) \rightarrow \pi_{1}(W)$ is an isomorphism. The above properties of $W$ are, however, not sufficient to guarantee that $W$ is the interior of a compact manifold. (See [5].)

The following is the main result.

Theorem 1. Suppose $W^{n}$ is a PL manifold which has homotopy type $K(\Pi, 1), W$ has one end $\infty, \pi_{1}$ is essentially constant at $\infty$, and the induced homomorphism $\pi_{1}(\infty) \rightarrow \pi_{1}(W)$ is an isomorphism. Then $\widetilde{W}$ is PL homeomorphic to $R^{n}$ provided $n \geq 5$.

Before proceeding with the proof of Theorem 1, we first make an application of Theorem 1. Let $I$ denote a countable group with finite cohomological dimension, denoted $\operatorname{cd}(\Pi)$. In [3] it is shown that if $\Pi$ is countable and $\operatorname{cd}(\Pi)$ is finite, then there is a polyhedron $X^{n}$ of type $K(\Pi, 1)$ of dimension $n=\operatorname{cd}(I)$ if $\operatorname{cd}(\Pi) \neq 2$, and of dimension $n=3$ if $\operatorname{cd}(I)=2$. Next it is shown that $X^{n}$ is homotopy equivalent to a subpolyhedron $Y^{n}$ of $R^{2 n}$. Let $W$ denote the interior of a regular neighborhood of $Y$. When $\operatorname{cd}(\Pi) \geq 2, Y$ has codimension $\geq 3$ in $W$ and therefore $W$ has one end $\infty, \pi_{1}$ is essentially con- 
stant at $\infty$, and $\pi_{1}(\infty) \rightarrow \pi_{1}(W)$ is an isomorphism. We have the following generalization of Corollary 3 of [3].

Theorem 2. Let $\Pi$ be a countable group of finite cohomological dimension $n$. If $n \neq 2$, there is a covering action of $\Pi$ on $R^{2 n}$, and if $n=2$ there is a covering action of $\Pi$ on $R^{6}$.

Proof. If $n \geq 2$ the theorem follows from preceding remarks together with Theorem 1. If $n=1$, this is Theorem 2 of [3].

All work is done in the PL category. [2] and [8] form standard references. Int and $\partial$ are used to denote interior and boundary, respectively, a superscript is used to denote dimension and " $\simeq$ " denotes "is PL homeomorphic to".

2. First we prove two lemmas.

Lemma 1. Suppose $W^{n}, n \geq 5$, is a PL manifold which has homotopy type $K(\Pi, 1), W$ has a single end $\infty, \pi_{1}$ is essentially constant at $\infty$, and the induced homomorphism $\pi_{1}(\infty) \rightarrow \pi_{1}(W)$ is an isomorphism. Then given a compact set $C$ in $W$, there is a compact set $D$ containing $C$ such that $\pi_{i}(W, W-D)=0$ for $i=0,1,2$.

Proof. From [5, Proposition 1.9, Part A], we have the existence of an arbitrarily small connected neighborhood $V$ of $\infty$, such that in the following commutative diagram

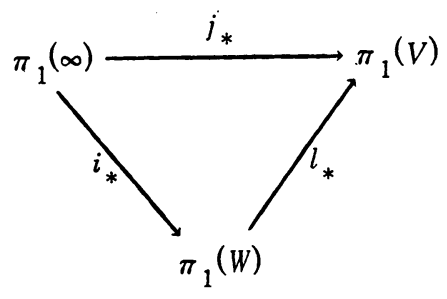

$j_{*}$ is an isomorphism by construction, $i_{*}$ is assumed to be an isomorphism, hence $l_{*}$ is an isomorphism. : But $\pi_{2}(W)=0$ and from the homotopy sequence of the pair $(W, V)$, we have $\pi_{i}(W, V)=0$ for $i=0,1,2$. By the definition of neighborhood of $\infty, W-V$ is compact. Therefore given a compact set $C$, let $V$ be a connected neighborhood of $\infty$ such that $V \cap C=\varnothing$; and such that $\pi_{1}(\infty) \rightarrow \pi_{1}(V)$ is an isomorphism. Then $W-V$ is compact, contains $C$, and $\pi_{i}(W, V)=0$ for $i=0,1,2$.

Lemma 2. Let $K$ be a triangulation of the manifold $W$. Let $J$ denote 
the i-skeleton of $K$, and $L$ the dual $(n-i-1)$-skeleton of $K$. Let $C$ denote a compact subset of $W$ such that $C \cap L=\varnothing$.: Then there exists a finite subcomplex $J_{0}$ of $J$ and a homeomorphism $k$ of $W$ such that

(1) $\left.k\right|_{J \cup L}=1$,

(2) $k(U) \supset C$,

where $U$ denotes the simplicial neighborhood of $J_{0}$ in $K^{\prime \prime}$.

Proof. As usual let $N\left(J, K^{\prime \prime}\right)$ and $N\left(L, K^{\prime \prime}\right)$ denote simplicial neighborhoods of $J$ and $L$, respectively, in $K^{\prime \prime}$. Observe that since $L$ is the dual skeleton to $J, \partial\left(N\left(J, K^{\prime \prime}\right)\right)=\partial\left(N\left(L, K^{\prime \prime}\right)\right)$, and from the mapping cyclinder structure of derived neighborhoods,

$$
N\left(L, K^{\prime \prime}\right)-L \simeq \partial\left(N\left(L, K^{\prime \prime}\right)\right) \times[0,1) .
$$

Let

$$
Y=N\left(J, K^{\prime \prime}\right) \cup\left(N\left(L, K^{\prime \prime}\right)-L\right)
$$

But $C \cap L=\varnothing$, so $C \subset Y$, and since $C$ is compact, one can use the product structure on $N\left(L, K^{\prime \prime}\right)-L$ to find a homeomorphism $k: Y \rightarrow Y$ with compact support such that $k\left(N\left(J, K^{\prime \prime}\right)\right) \supset C$ and $k \mid J=1$. Extend $k$ by the identity to L. Let

$$
J_{0}=\left\{\sigma \in J: k^{-1}(C) \cap N\left(\sigma, K^{\prime \prime}\right) \neq \varnothing\right\}
$$

The compactness of $C$ implies $J_{0}$ is finite and from the definition of $J_{0}$, $k\left(N\left(J_{0}, K^{\prime \prime}\right)\right) \supset C$.

Proof of Theorem 1. From [1] it suffices to show that given a compact set $D$ contained in $\widetilde{W}, D$ is contained in the interior of an $n$-ball.

Let $K$ denote a triangulation of $W, \widetilde{K}$ the induced triangulation of $\widetilde{W}$.

Let $J$ denote the $(n-3)$-skeleton of $K$, and $L$ the dual 2-skeleton. Let $p$ denote the projection of $\widetilde{W}$ onto $W$ and let $C=p(D) . C$ is compact. By Lemma 1 , there is a neighborhood $V$ of $\infty$ such that

(1) $C \subset W-V$,

(2) $\pi_{i}(W, V)=0, i=0,1,2$.

From standard engulfing, as in [2], there is a homeomorphism $h: W \rightarrow W$

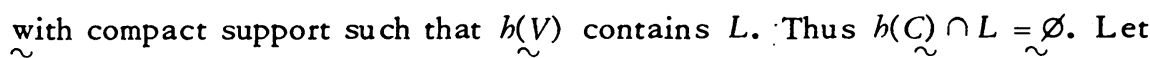
$\tilde{h}$ denote the homeomorphism on $\widetilde{W}$ which covers $h$. Then $\tilde{h}(D) \cap \tilde{L}=\varnothing$. Let $D^{\prime}=\tilde{h}(D)$. From Lemma 2 , one can find a finite $\underset{\sim}{\sim} \sim_{\sim}$ complex $\tilde{J}_{0}$ of $\tilde{J}$ and a homeomorphism $k: \widetilde{W} \rightarrow \underset{\widetilde{W}}{\sim}$ such that $k\left(N\left(\tilde{J}_{0}, \widetilde{K}^{\prime \prime}\right)\right) \supset D^{\prime}$. Now $\widetilde{W}$ is contractible, $\operatorname{dim} \tilde{J}_{0} \leq n-3$, hence $\tilde{J}_{0}$ lies in the interior of a ball. :But $N\left(\tilde{J}_{0}, \widetilde{K}^{\prime \prime}\right)$ collapses to $\tilde{J}_{0}$, hence $N\left(\tilde{J}_{0}, \tilde{K}^{\prime \prime}\right)$ lies in the interior of a ball. Let $B$ denote such a ball. Then $k(B) \supset D^{\prime}$ and $\widetilde{h}^{-1} k(B) \supset D$. 
The following is an outline of the construction of the 3-dimensional example promised in the introduction. Let $M^{3}$ denote an open $K(\Pi, 1)$ manifold, and let $W^{3}$ denote an open, contractible 3-manifold which does not embed in $R^{3}$ [4]. Let $L_{1}$ and $L_{2}$ denote the images of $[0, \infty)$ under PL embeddings in $M$ and $W$, respectively. Let $M^{\prime}$ and $W^{\prime}$ denote the complements in $M$ and $W$, respectively, of the interior of a regular neighborhood of each of $L_{1}$ and $L_{2}$. Then $\partial M^{\prime} \simeq \partial W^{\prime} \simeq R^{2}$. Let $N=M^{\prime} \cup_{b} W^{\prime}$, where $h$ is an orientation preserving homeomorphism from $\partial M^{\prime}$ onto $\partial W^{\prime}$. Then the universal cover $\tilde{N}$ of $N$ is an open contractible manifold and so $N$ is a $K(\Pi, 1)$ manifold. Also $W^{\prime} \subset N$ lifts to infinitely many disjoint copies of $W^{\prime}$ in $\widetilde{N}$. But int $W^{\prime}$ is homeomorphic to $W$ and so $\widetilde{N}$ cannot be homeomorphic to $R^{3}$.

\section{REFERENCES}

1. M. Brown, The monotone union of open n-cells is an open n-cell, Proc. Amer. Math. Soc. 12 (1961), 812-814. MR 23 \# A4129.

2. J. F. P. Hudson, Piecewise linear topology, Univ. of Chicago Lecture Notes, Benjamin, New York, 1969. MR 40 \#2094.

3. F. E. A. Johnson, Manifolds of homotopy type $K(\pi, 1)$, Proc. Cambridge Philos. Soc. 70 (197 1), 387-393. MR 44 \#7542.

4. J. M. Kister and D. R. McMillian, Jr., Locally euclidean factors of $E^{4}$ which cannot be imbedded in E3, Ann. of Math. (2) 76 (1962), 541-546. MR 26 \#1868.

5. L. C. Siebenmann, On detecting open collars, Tran s. Amer. Math. Soc. 142 (1969), 20 1-227. MR 39 \#7605.

6. - Doctoral Dissertation, Princeton University, Princeton, N. J., 1965.

7. F. Waldhausen, On irreducible 3-manifolds which are sufficiently large, Ann. of Math. (2) 87 (1968), 56-88. MR 36 \#7146.

8. E. C. Zeeman, Seminar on combinatorial topology, Inst. Hautes Etudes Sci., Paris, 1963, 1966 (mimeographed notes).

DEPARTMENT OF MATHEMATICS, OKLAHOMA STATE UNIVERSITY, STILLWATER, OKLAHOMA 74074 